\title{
过渡金属催化的 C-P 键活化
}

\author{
张凤萍栾玉新* 叶萌春* \\ (南开大学化学学院 元素有机化学国家重点实验室 天津 300071)
}

\begin{abstract}
摘要 过渡金属催化的 $\mathrm{C}-\mathrm{P}$ 键活化转化为有机膦化合物的合成提供了一条经济、高效的途径, 近年来受到了越来越多 的关注. 然而由于 $\mathrm{C}-\mathrm{P}$ 键较高的键能和 $\mathrm{P}$ 原子强的配位性能, $\mathrm{C}-\mathrm{P}$ 键活化一直较为挑战. 采用不同的底物预活化方法 和各类催化剂, 过渡金属催化的 $\mathrm{C}-\mathrm{P}$ 键活化转化已经取得了显著的进展. 本综述将对该领域的发展进行总结, 按照反 应机理和底物预活化策略的不同，分类阐述这些反应的类型，发展、特点和机理等.
\end{abstract}

关键词 有机膦化合物; 过渡金属催化; $\mathrm{C}-\mathrm{P}$ 键活化

\section{Transition Metal-Catalyzed C-P Bond Activation}

\author{
Zhang, Fengping Luan, Yuxin* Ye, Mengchun* \\ (State Key Laboratory and Institute of Elemento-organic Chemistry, College of Chemistry, Nankai University, Tianjin 300071)
}

\begin{abstract}
Transition metal-catalyzed $\mathrm{C}-\mathrm{P}$ bond activation provides an ecomomical and high-efficient route for the synthesis of organic phosphine compounds, and has received increasing attention in recent years. Owing to high bond energy of $\mathrm{C}-\mathrm{P}$ bond and strong coordinative ability of $\mathrm{P}$ atom, the activation of $\mathrm{C}-\mathrm{P}$ bond has been a fomidable challenge. Relying on substrate-preactivation and various catalysts, great progress has been achieved. This review will give a summary of this field, and according to different mechanisms of $\mathrm{C}-\mathrm{P}$ bond activation and strategies of substrate pre-activation, a detailed description on reaction type, development, characteristics and mechanism will be made.
\end{abstract}

Keywords organophosphines; transition metal; $\mathrm{C}-\mathrm{P}$ bond activation

有机膦化合物广泛存在于生物活性分子和材料分 子中, 具有重要的生理活性和光电性能 ${ }^{[1]}$. 此外, 有机 膦化合物广泛应用于有机合成领域, 不仅可以作为多样 性金属配体或者催化剂, 促进各种有机合成反应, 而且 自身也是重要的合成试剂, 可以参与多种类型的有机合 成反应, 如 Witting 试剂参与的烯化反应, Lawesson 试剂 参与的氧硫交换反应等 ${ }^{[2]}$. 因此, 发展经济、高效的有机 膦合成方法具有重要的研究意义和应用价值, 长久以来 受到了广泛而持续的关注.

传统的有机膦化合物的合成主要依赖无机含磷试 剂的转化, 如 $\mathrm{P}_{4} 、 \mathrm{PCl}_{3} 、 \mathrm{PH}_{3}$ 等, 不仅需要较长的反应步 骤, 而且这类试剂通常稳定性差, 不易储存和应用, 且 反应往往需要活泼的金属试剂和剧烈的反应条件, 产生 等物质的量的废物, 难以获得高的原子和步骤经济性 ${ }^{[3]}$.
为此, 直接利用有机膦化合物作为原料, 通过 $\mathrm{C}-\mathrm{P}$ 键 的切断来构建有机膦化合物是一个较为经济和直接的 选择, 因为有机膦化合物结构多样, 且很多已经实现了 规模化生产, 可以提供范围广泛的底物选择. 目前, 已 有多种方法被用于 $\mathrm{C}-\mathrm{P}$ 键的切断和重组, 包括过渡金 属催化 ${ }^{[4]}$ 、光促进 ${ }^{[5]}$, 以及其他试剂介导的方法 ${ }^{[6]}$. 其中, 通过过渡金属催化的 $\mathrm{C}-\mathrm{P}$ 键活化构建新有机膦化合物 受到了最为广泛的重视. 该方法的优势体现在以下两个 方面: (1)直接过渡金属催化转化的方法可以利用成熟的 有机膦结构, 进行快速衍生化; 同时, 可以实现有机膦 化物的后期修饰, 避免繁琐的保护和脱保护步骤, 大大 地缩短合成步骤, 提高反应的原子和步骤经济性; (2)利 用多样性的配体调控, 有望实现此类转化的选择性调 控, 获得高的化学选择性、位点选择性和立体选择性.

\footnotetext{
* Corresponding authors. E-mail: yxluan@nankai.edu.cn; mcye@nankai.edu.cn Received May 31, 2021; revised July 12, 2021; published online July 23, 2021. Dedicated to the 100th anniversary of Chemistry at Nankai University.

Project supported by the National Natural Science Foundation of China (Nos. 21871145, 91856104) and the Natural Science Foundation of Tianjin City (No. 19JCZDJC37900).

国家自然科学基金(Nos. 21871145, 91856104)和天津市自然科学基金(No. 19JCZDJC37900)资助项目.
} 
过渡金属催化的 $\mathrm{C}-\mathrm{H} 、 \mathrm{C}-\mathrm{C} 、 \mathrm{C}-\mathrm{O} 、 \mathrm{C}-\mathrm{N}$ 等的 活化转化均已被广泛研究 ${ }^{[7-10]}$, 然而, $\mathrm{C}-\mathrm{P}$ 键的活化转 化研究相对贵乏. 主要的困难在于: 一方面, C-P 的键 能和 $\mathrm{C}-\mathrm{N}$ 的键能类似, 都是较为惰性的化学键, 不易 活化; 另一方面, $\mathrm{P}$ 原子具有更大的电子密度, 容易与过 渡金属产生较强的配位作用，抑制了金属对 $\mathrm{C}-\mathrm{P}$ 键的 活化. 尽管如此, 基于化学计量的金属对 $\mathrm{C}-\mathrm{P}$ 键活化 的研究经验, 人们认识到采用不同的 $\mathrm{C}-\mathrm{P}$ 键活化机理 和合适的底物预活化方式, 可以实现过渡金属催化的 $\mathrm{C}-\mathrm{P}$ 键的活化转化, 并可以高效、简洁地构建各类有机 膦化合物. 目前已有多篇综述介绍了过渡金属催化的 $\mathrm{C}-\mathrm{P}$ 活化及偶联反应 ${ }^{[4]}$, 但是对于各类 $\mathrm{C}-\mathrm{P}$ 键活化反 应的机理和策略尚缺乏系统的归纳和分类. 为此, 我们 将在此篇综述中对该领域的进展进行详细归类和评述. 根据 $\mathrm{C}-\mathrm{P}$ 键活化方式的不同, 各类 $\mathrm{C}-\mathrm{P}$ 键活化反应大 致可以分为两类: 第一, 通过 $\mathrm{C}-\mathrm{P}$ 键和过渡金属的直 接氧化加成途径实现 $\mathrm{C}-\mathrm{P}$ 键活化; 第二, 通过过渡金 属诱导的极性键的加成消去途径实现 $\mathrm{C}-\mathrm{P}$ 键活化. 对 于氧化加成途径, 为了获得更高的反应活性, 多数底物 往往需要额外的预活化. 按照活化的强弱程度不同, 我 们还将此类途径进一步细分为五个子类, 包括: (1)季鏻 盐促进 C-P 键活化; (2)酰基膦促进 $\mathrm{C}-\mathrm{P}$ 键活化; (3)环 张力促进 $\mathrm{C}-\mathrm{P}$ 键活化; (4) 导向基促进 $\mathrm{C}-\mathrm{P}$ 键活化; (5) 催化剂促进 $\mathrm{C}-\mathrm{P}$ 键活化.

\section{C 一 P 键和过渡金属的直接氧化加成途径}

共价键与低价过渡金属的氧化加成, 是过渡金属催 化活化共价键的常见途径. 基于此途径的各类惰性键的 活化, 像 $\mathrm{C}-\mathrm{H} 、 \mathrm{C}-\mathrm{C}$ 或 $\mathrm{C}-\mathrm{N}$ 等键的活化, 已得到广 泛研究. 这类惰性键活化途径的一般规律是, 较为缺电 子的共价键更易于和较为富电子的低价金属进行氧化 加成. 然而, 由于 $\mathrm{C}-\mathrm{P}$ 键通常具有较高的电子云密度 和较高的键能, 更难发生与金属的氧化加成. 针对这一 难点, 最为常见的方法是通过底物的预活化, 来降低 $\mathrm{C}-\mathrm{P}$ 键的电子密度, 从而促进与金属的氧化加成. 根 据底物活化方式的不同, 将按照以下五个类型加以介 绍.

\section{1 季鏻盐促进 C一P 键活化}

通过将有机膦化合物转化为季鏻盐, 可以使 $\mathrm{P}$ 原子 带有较强的正电性, 从而显著减弱 $\mathrm{C}-\mathrm{P}$ 键的键能, 利 于和金属的氧化加成. 根据季鏻盐的形成方式不同, 这 类体系又可分为两类: (1)预先制备的季鏻盐底物, (2)原 位生成的季鏻盐底物. 前者需要直接预先合成含有正电 性 $\mathrm{P}$ 的季鏻盐底物，后者则由过渡金属辅助，现场生成 季鏻盐底物.
1.1.1 季鏻盐关键中间体的发现和金属络合物的研 究

1992 年, Marcuccio 等 ${ }^{[11]}$ 在研究 $\mathrm{Pd}\left(\mathrm{Ph}_{3} \mathrm{P}\right)_{4}$ 催化的烷 氧基取代芳基硼酸和烷氧基取代的芳基溴的 Suzuki 偶 联反应时，检测到了芳基嗍酸的苯基化副产物(图 1). 由 于无论使用苯还是 1,2-二甲氧基乙烷为溶剂, 均会得到 该副产物，且不使用溴代芳烃的控制实验仍然得到了该 副产物，于是作者推测副产物的苯基来自配体. 为了进 一步证实该推测，作者进行了相关的控制实验，发现通 过降低催化剂用量和使用更加富电子的芳基磷配体，可 以显著降低该副产物的生成. 据此，作者认为副产物的 生成是钯中间体的芳基-芳基交换所致。这是最早的关 于 $\mathrm{Pd}-\mathrm{P}$ 之间配体交换的讨论.

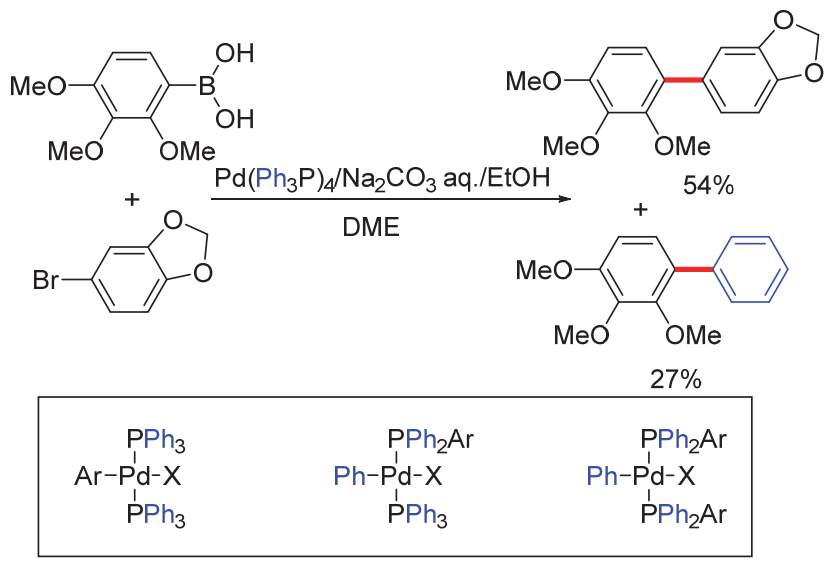

图 $1 \mathrm{Pd}$ - $\mathrm{P}$ 之间的配体交换现象

Figure 1 Ligand exchange between $\mathrm{Pd}$ and $\mathrm{P}$

最初，这类过渡金属和配位的有机膦化合物之间的 基团交换被认为经历了直接的基团迁移过程．直到 1995 年, Yamamoto 等 ${ }^{[12]}$ 提出了一种新的基团迁移路径, 即芳基卤在 $\mathrm{P}$ 中心的还原消除生成季鏻盐，随后 $\mathrm{Pd}(0)$ 再和 $\mathrm{Pd}-\mathrm{P}$ 键进行氧化加成(图 2).

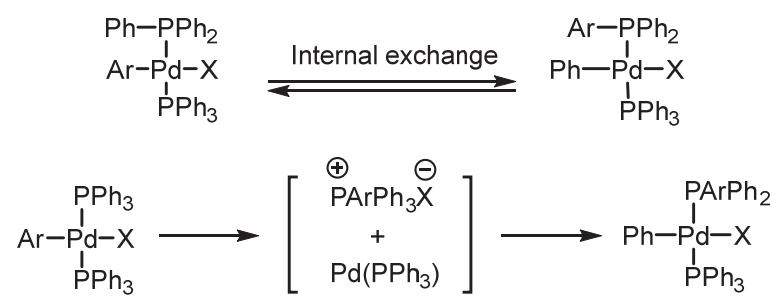

图 2 季鏻盐关键中间体的提出

Figure 2 Proposed quaternary-phosphonium-salt key intermediate

2004 年, Glueck 等 ${ }^{[13]}$ 进行手性膦配体的反应性能研 究时, 发现四元碳一磷杂环可以进行 $\mathrm{C}-\mathrm{P}$ 键裂解, 得到 $\mathrm{P}$-烷基/Pd-苯基交换的环，从而可以很容易地转化为带 
有 $\mathrm{P}$ 和 $\mathrm{C}$ 立体中心的新型双齿配体的络合物(图 3). 令 人惊讶的是, 在温和的条件下, 钯介导的膦烷开环的同 时, 苯基从钯迁移到膦. 从对 $\mathrm{P}-\mathrm{Me} / \mathrm{Pd}-\mathrm{Ph}$ 交换不可 逆性的观察来看, Norton 等 ${ }^{[14]}$ 推测 $\mathrm{Pd}-\mathrm{Ph}$ 和 $\mathrm{P}-\mathrm{Me}$ 键 总体上比 $\mathrm{Pd}-\mathrm{Me}$ 和 $\mathrm{P}-\mathrm{Ph}$ 键更强.

2006 年, Landis 等 ${ }^{[15]}$ 进一步发现具有 5 元环结构的 膦烷基也可以和金属烷基进行交换: 首先, [(cod)Pd$(\mathrm{Me}) \mathrm{Cl}$ 与与底物的 $\mathrm{P}$ 配位; 随后发生基团迁移, 甲基迁移 到 P 上, 同时二氮杂膦环开环(图 4). 尽管作者没有进行 机理推测, 但考虑到反应和 Glueck 等[13]发现的反应具 有高度的相似性, 我们推测该反应应该同样涉及季鏻盐 中间体.

2012 年, Mecking 等 ${ }^{[16]}$ 发现磺酸基辅助的 Pd 络合物 中, $\mathrm{Pd}-\mathrm{Me}$ 与 $\mathrm{P}-\mathrm{Ar}$ 可以发生基团交换(图 5). 作者推 测, 络合物中取代基的交换是通过季鏻盐关键中间体进 行的.
在芳基亲电试剂参与的偶联反应中, $(R)-(+) 2,2^{\prime}-二$ (二苯基膦)-1, $1^{\prime}$-二䒺(BINAP)衍生物侧臂的芳基交换是

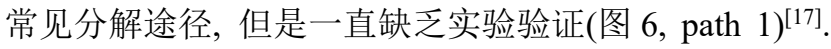
为此, 2014 年, Hartwig 等 ${ }^{[18]}$ 进行了相关的研究. 结果显 示，在含有 BINAP 和芳基卤化物的 $\mathrm{Ni}$ 或 Pd 络合物的反 应过程中，配体主链发生了 $\mathrm{P}-\mathrm{C}$ 键断裂。作者推测该 反应同样通过季鏻盐中间体进行(图 6,path 2).

2014 年, Braunstein 课题组 ${ }^{[19]}$ 报道了另外一例 P 导 向的 $\mathrm{P}-\mathrm{Ar} / \mathrm{Pd}-\mathrm{Me}$ 交换反应(图 7). 底物中, 一个二苯 基膦和一个二叔丁基膦同时连接在咪唑环上，但是只有 二芳基膦与甲基 $\mathrm{Pd}$ 发生基团交换.

2018 年, $\mathrm{Rh}$ 催化剂也被用于 $\mathrm{C}-\mathrm{P}$ 键活化研究(图 $8)^{[20]}$. 在含有卡宾和烷基膦的双齿导向基的辅助下，金 属 $\mathrm{Rh}$ 进行还原消除生成季鏻盐中间体，然后 $\mathrm{Rh}$ 金属再 和 $\mathrm{Ar}-\mathrm{P}$ 键进行氧化加成, 从而可以在相对温和的条件 下活化 $\mathrm{C}-\mathrm{P}$ 键.

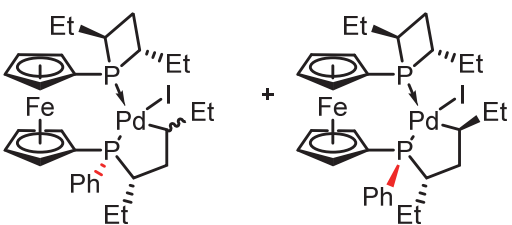

图 3 四元磷环和芳基钯的开环反应

Figure 3 Ring-opening reaction of 5-membered P-ring with arylpalladium<smiles></smiles><smiles>C[Ge]1(Cl)CCCCC1</smiles>

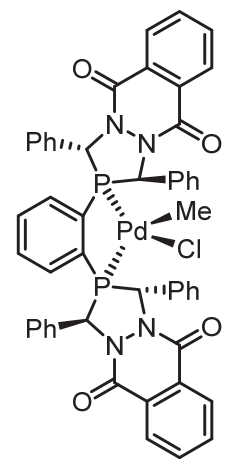

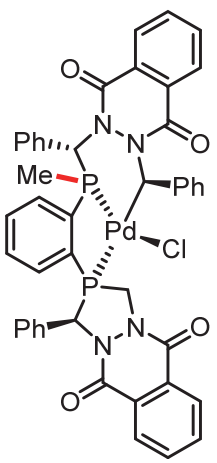

图 4 五元膦环和甲基钯的开环反应

Figure 4 Ring-opening reaction of 5-membered P-ring with methylpalladium

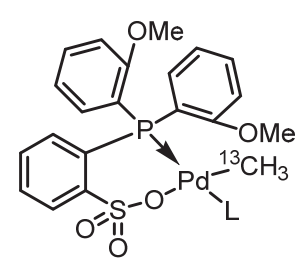<smiles>COc1ccccc1[PH](C)(c1ccccc1OC)c1ccccc1S(=O)(=O)[O-]</smiles><smiles>COc1ccccc1[P@]1(C)c2ccccc2S(=O)(=O)O[P+]1(C)c1ccccc1OC</smiles>

图 5 磺酸基导向的 $\mathrm{Pd}-\mathrm{Me}$ 与 $\mathrm{P}-\mathrm{Ar}$ 基团交换

Figure 5 Sulfonate-directed group exchange between $\mathrm{Pd}-\mathrm{Me}$ and $\mathrm{P}-\mathrm{Ar}$ 


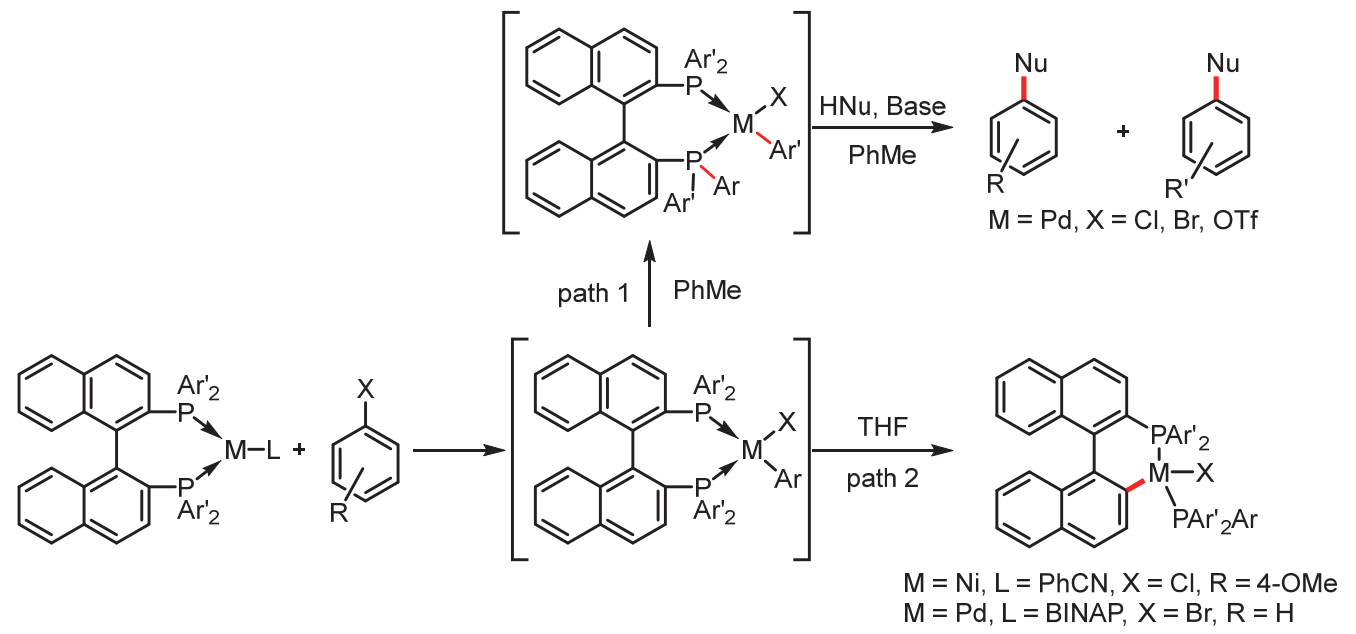

图 6 BINAP 的分解途径

Figure 6 Decomposition pathways of BINAP

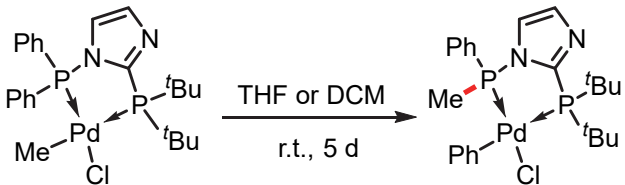

图 $7 \mathrm{P}$ 导向的 $\mathrm{Pd}-\mathrm{Me}$ 与 $\mathrm{P}-\mathrm{Ar}$ 基团交换

Figure 7 P-directed group exchange between $\mathrm{Pd}-\mathrm{Me}$ and $\mathrm{P}-\mathrm{Ar}$

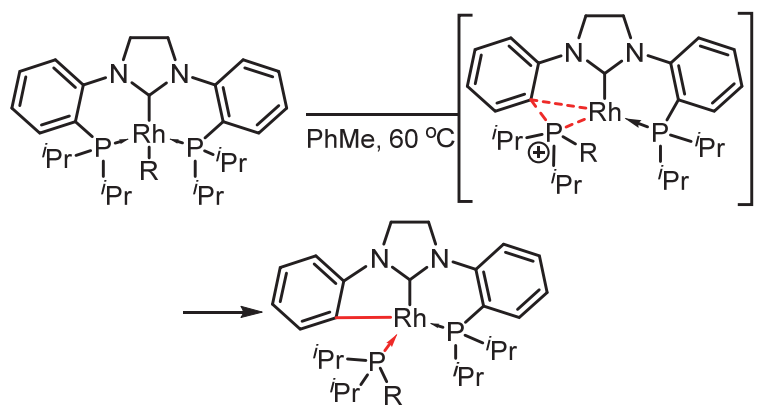

图 8 双齿导向基促进的 $\mathrm{C}-\mathrm{P}$ 键活化

Figure 8 Bidentate group-enabled $\mathrm{C}-\mathrm{P}$ bond activation

\subsection{2 预先制备的季鏻盐底物}

1995 年, Yamamoto 等 ${ }^{[12]}$ 基于他们提出的季鏻盐关 键中间体，预先制备了各类季鏻盐，发现它们可以顺利 进行钯催化的烯基化和羰基化反应，且反应的活性不受 卤素阴离子的影响, 明显不同于卤代芳烃底物参与的偶 联反应. 此外, 季鏻盐还可以参与加氢反应, 为从简单 的三苯基膦制备其他更为复杂取代的膦化合物提供了 一个简便的方法(图 9).

2004 年, Vicente 等 ${ }^{[21]}$ 进行了季鏻盐的性质研究, 发 现 $\left[\mathrm{Ph}_{4} \mathrm{P}\right][2-\mathrm{BrPhS}]$ 与零价 $\mathrm{Pd}$ 在 $\mathrm{PPh}_{3}$ 存在下, 可以得到 硫芳基化的钯络合物. 作者推测, 反应中 $\operatorname{Pd}(0)$ 先与季 鏻盐的 $\mathrm{P}-\mathrm{Ph}$ 氧化加成, 而不是先与 $\mathrm{C}-\mathrm{Br}$ 键氧化加成 (图 10).

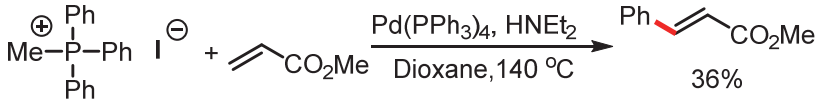

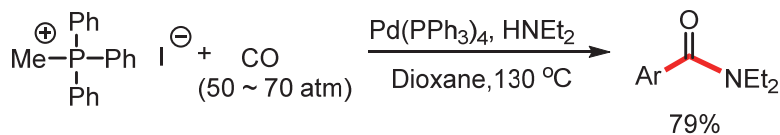

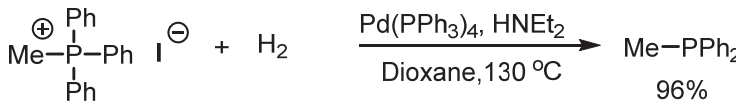

图 9 钯催化的季鏻盐转化

Figure 9 Palladium-catalyzed transformation of phosphonium salts
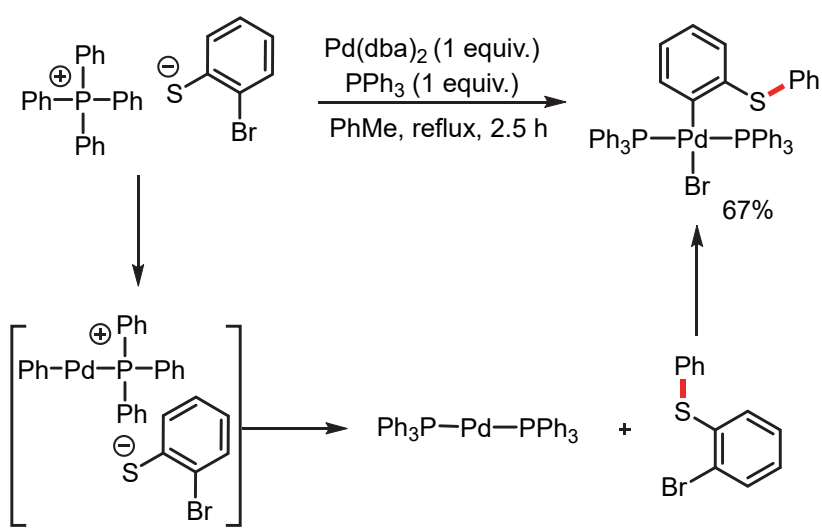

图 10 季鏻盐 $\mathrm{Ph}-\mathrm{P}$ 和 $\mathrm{Ar}-\mathrm{Br}$ 键的竞争性氧化加成 Figure 10 Competitive oxidative addition between $\mathrm{P}-\mathrm{Ph}$ and $\mathrm{Ar}-\mathrm{Br}$ bond

2005 年, 通过优化反应条件, Chang 等 ${ }^{[22]}$ 实现了 Pd 催化季鏻盐的 Heck 偶联反应(图 11). 以 $\mathrm{Pd}(\mathrm{OAc})_{2}$ 为催 化剂, $\mathrm{NaOAc}$ 为碱，丙烯酸酯、丙烯酰胺、丙烯腈等多 种烯基化试剂均可以参与和 $\mathrm{Ph}_{4} \mathrm{P}^{+} \mathrm{Cl}^{-}$的反应，以中等至 优异的收率得到苯基烯基化产物. 值得说明的是，季鏻 盐的抗衡阴离子对反应收率有较为明显的影响, $\mathrm{Cl}^{-}$相 
较于 $\mathrm{Br}^{-}$和 $\mathrm{I}^{-}$有更高的活性. 此外, 反应在空气氛围中 进行比氮气氛围中通常高出 $20 \%$ 的收率. 该作者认为, 氧气可以将游离的三苯基膦氧化为膦氧, 从而有效促进 反应的进行. 除 Heck 烯烃化, 四芳基卤化鏻也可以顺 利参与 Suzuki 和 Sonogashira 反应. 值得一提的是, 当四 芳基卤化鏻的芳基取代基不同时，不管是含有供电子或 吸电子的取代基，反应均会得到混合物，暗示了芳基在 $\mathrm{P}$ 和 Pd 之间交换较为快速, 受电子因素的影响较小.

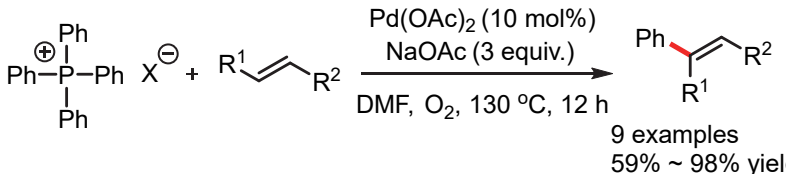

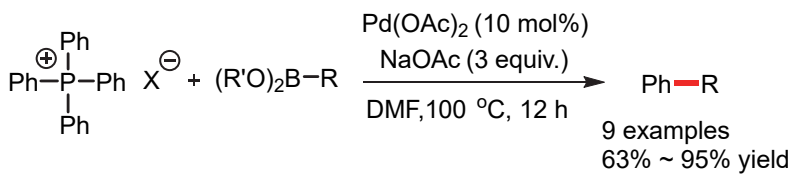

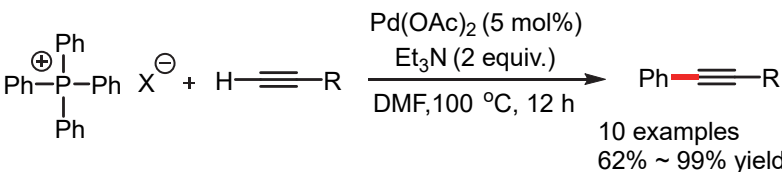

图 11 钯催化分子间芳基化反应

Figure 11 Palladium catalyzed intermolecular arylation

为了解决含有不同芳基取代基季鏻盐的基团迁移 的选择性问题, 2017 年, McNally 等 ${ }^{[23]}$ 设计合成了含杂 芳环的季鏻盐, 发现它们与芳基硼试剂反应时, 可以成 功实现杂芳环的选择性迁移 (图 12). 所使用的杂芳环为 在药物中广泛存在的吡啶和二嗪, 相应的季鏻盐可以方 便地从卤代的吡啶或二嗪与 $\mathrm{Ph}_{3} \mathrm{P}$ 的直接反应获得. 该 方法为 4-取代吡定的选择性合成提供了一种高效的方 法.

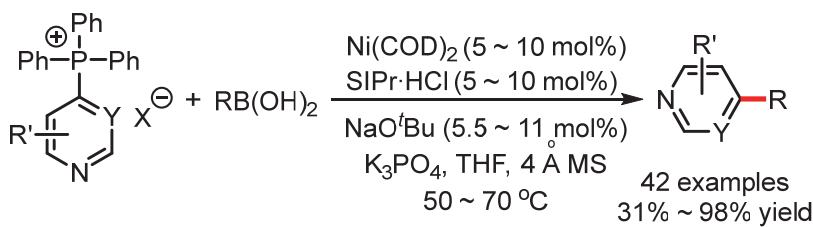

图 12 镍催化的分子间芳基化

Figure 12 Nickel-catalyzed intermolecular arylation

\section{1 .3 原位生成的季鏻盐底物}

不同于预先生成的季鏻盐, 在反应过程中原位生成 季鏻盐中间体通常经历卤代物对金属催化剂的氧化加 成、含 $\mathrm{P}$ 化合物对高价金属配位、还原消除等步骤.

1995 年, Chenard 等 ${ }^{[24]}$ 在钯催化的 Stille 反应中发现 溴代烃芳基可以与 $\mathrm{Ph}_{3} \mathrm{P}$ 配体的苯基发生交换反应. 当 使用 $5 \mathrm{~mol} \%$ 的 $\left[\mathrm{Pd}\left(\mathrm{PPh}_{3}\right)_{4}\right]$ 时, 可以得到 $55 \%$ 的副产物
(图 13). 该芳基交换副反应受溶剂影响较小，且使用缺 电子的卤代烃可以明显降低竞争性副反应的发生. 基于

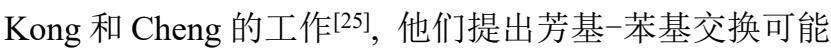
经历季鏻盐中间体的机理设想, 并通过 $\mathrm{Ph}_{4} \mathrm{P}^{+} \mathrm{Br}^{-}$的对 照实验证实了这一假设. 后来, Novak 等 ${ }^{[26]}$ 通过 $\mathrm{Ar}^{\prime} \mathrm{PdPAr}_{3} \mathrm{I}$ 的机理实验进一步阐释了鏻盐的作用，以及 膦配体对芳基-苯基交换的电子和空间效应.

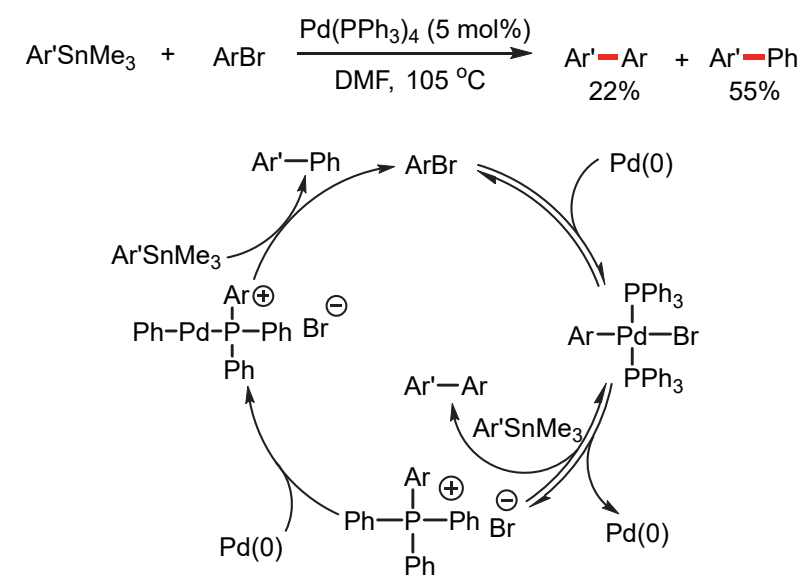

图 13 钯催化 Stille 偶联反应中的基团交换

Figure 13 Group exchange in Palladium-catalyzed Stille coupling reaction

2000 年, 基于 $\mathrm{Pd}-\mathrm{Ar} / \mathrm{P}-\mathrm{Ar}$ 交换的反应性, Chan 等 ${ }^{[27]}$ 以三芳基膦和不同取代的溴代芳烃为底物, 制备 了不同取代的三芳基膦化合物(图 14), 为膦配体的高效 合成提供了一条便捷的途径. 反应具有较好的官能团兼 容性，含酮、醛、酯、醚和卤素等的各类官能团的底物 均可很好地参与反应.

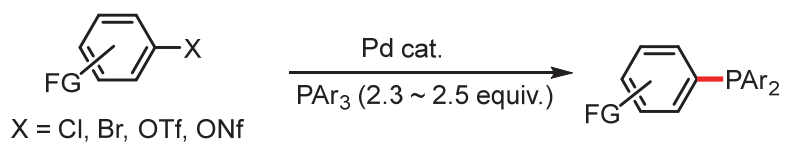

图 14 钯催化卤代芳烃和三芳基膦的膦化反应

Figure 14 Pd-catalyzed phosphination reaction of aryl halides with triarylphosphines

2013 年, Chatani 等 ${ }^{[28]}$ 利用 C-P 键在金属中心的还 原消除可以原位形成季鏻盐中间体的性质，发展了一种 五元环三芳基膦化合物的合成方法(图 15). 该反应具有 原料价廉易得，反应易于操作，和官能团兼容性好等优 点, 为合成新的三芳基膦配体提供了一种高效的方法.

作者推测该反应的机理如下: $\mathrm{P}$ 原子导向钯 $\mathrm{C}-\mathrm{H}$ 活化后得到六元环中间体，而后还原消除形成五元环季 鏻盐. 该季鏻盐的 $\mathrm{C}-\mathrm{P}$ 键与 $\mathrm{Pd}(0)$ 进行氧化加成, 再经 历质解和还原消除得到三芳基膦产物.

2015 年, Chatani 课题组 ${ }^{[29]}$ 进一步发展了分子内 C$\mathrm{P}$ 键活化与 $\mathrm{C}-\mathrm{Br}$ 键偶联的成环反应，同样提供了一种 


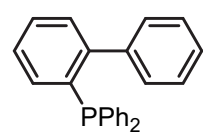

$\mathrm{Pd}(\mathrm{OAc})_{2}(5 \mathrm{~mol} \%)$ Toluene, $160^{\circ} \mathrm{C}$

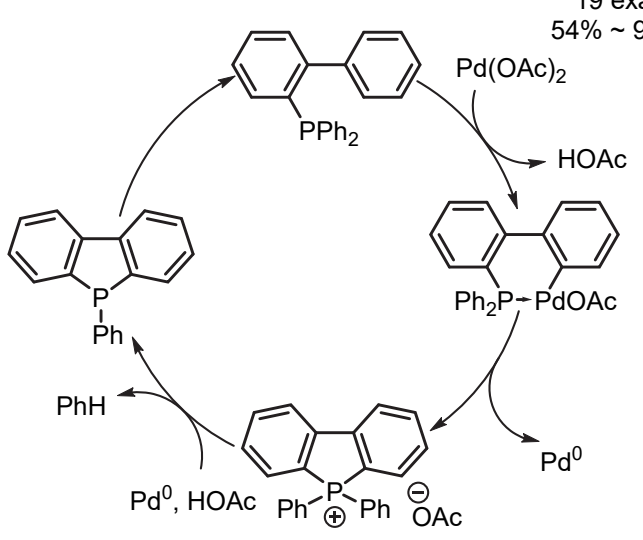

图 15 钯催化分子内 $\mathrm{C}-\mathrm{H}$ 膦化反应

Figure 15 Pd-catalyzed intramolecular $\mathrm{C}-\mathrm{H}$ phosphination

新型膦化合物的合成方法(图 16). 作者提出的机理与之 前所报道的体系类似，但需要 $\left(\mathrm{Me}_{3} \mathrm{Si}\right)_{3} \mathrm{SiH}$ 作为还原剂 将二价钯转化为活性零价钯.

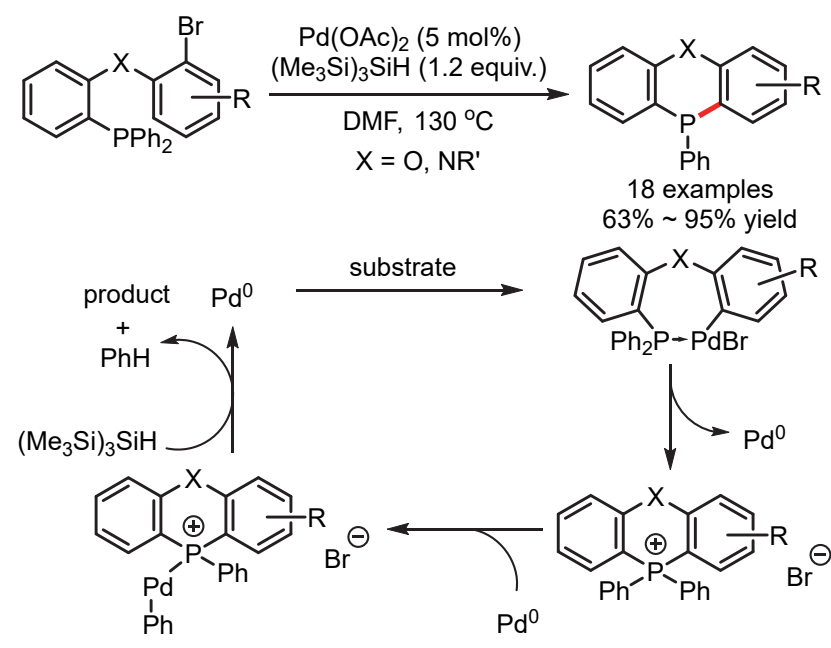

图 16 钯催化分子内 $\mathrm{C}-\mathrm{Br}$ 膦化反应

Figure 16 Pd-catalyzed intramolecular $\mathrm{C}-\mathrm{Br}$ phosphination

同年, 蒋高喜和夏春谷等 ${ }^{[30]}$ 报道了 $\mathrm{Pd}(\mathrm{OTf})_{2}$ 催化 1,4-二氢-1,4-环氧䒺与叔膦的芳基化反应(图 17). 该反 应使用 $\mathrm{Cu}(\mathrm{OAc})_{2}$ 作为氧化剂, 可以顺利地得到一系列 2-芳基萗产物. 含不同取代基的三取代膦的竞争实验显 示, 苯基优先于烷基发生迁移, 缺电子的芳基比富电子 的芳基更易迁移.

反应后分离得到二苯基膦酸 $\left(\mathrm{Ph}_{2} \mathrm{PO}_{2} \mathrm{H}\right)$, 表明三苯 基膦的含膦部分基团最终被氧化了. 据此, 作者提出了 可能的机理如下: $\mathrm{Pd}(\mathrm{II})$ 与 $\mathrm{PPh}_{3}$ 作用得到了关键活性化 合物 $\mathrm{Pd}-\mathrm{A}$. 随后, $\mathrm{Pd}-\mathrm{A}$ 与底物烯烃进行迁移插入, 生 成中间体 $\mathrm{Pd}-\mathrm{B}$, 进一步 $\beta$-O-消除生成 $\mathrm{Pd}-\mathrm{C}$. 最后,

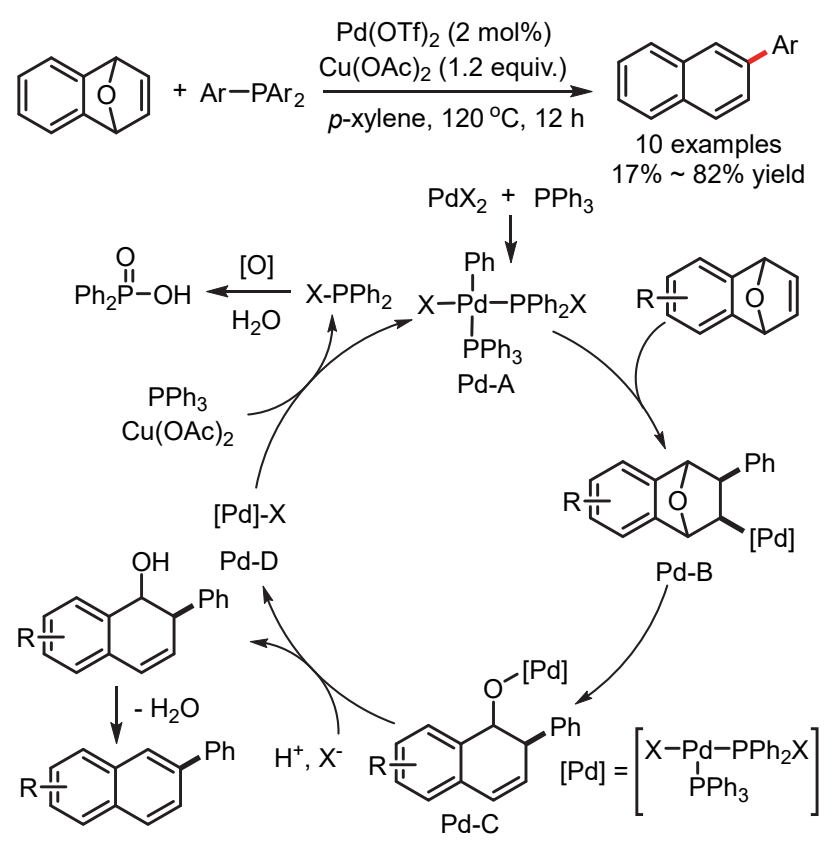

图 17 三芳基膦作为钯催化 Heck 型反应的芳基化试剂 Figure $17 \quad \mathrm{Ar}_{3} \mathrm{P}$ as arylating reagent in Pd-catalyzed Heck-type reaction

$\mathrm{Pd}-\mathrm{C}$ 经酸解后释放出醇化合物和 $\mathrm{Pd}-\mathrm{D}$. 醇化合物脱 水芳构化得到所需产物, 而 $\mathrm{Pd}-\mathrm{D}$ 再与 $\mathrm{PPh}_{3}$ 和 $\mathrm{Cu}(\mathrm{OAc})_{2}$ 作用生成 $\mathrm{Pd}-\mathrm{A}$ 和 $\mathrm{Ph}_{2} \mathrm{P}-\mathrm{X}$. 后者经过氧化得 到二苯基膦酸.

2016 年, 王智谦课题组 ${ }^{[31]}$ 报道了酰基膦与芳基卤 代物的分子间脱羰偶联反应(图 18). 该反应以 $\mathrm{Pd}(\mathrm{OAc})_{2}$ 作为催化剂, 碳酸铯作为碱, 甲苯作为溶剂, 在 $80{ }^{\circ} \mathrm{C}$ 下得到偶联产物。作者推测该反应的可能机理如下：醋 酸钯与酰基膦配位, 随后, 零价钯与碘苯氧化加成形成 二价钯; 经过还原消除得到 $\mathrm{Pd}^{0}$ 和关键的季鏻盐中间体; 接着, $\mathrm{Pd}^{0}$ 与酰基膦的 $\mathrm{C}-\mathrm{P}$ 键发生氧化加成, 并脱羰, 还原消除形成芳基膦偶联化合物.

2017 年, Morandi 等 ${ }^{[32]}$ 报道了钯催化双膦底物的分 子内膦化反应，得到一系列三芳基单膦化合物的反应 (图 19). 反应需要加入 $10 \mathrm{~mol} \%$ 的 $\mathrm{PhI}$ 作为助催化剂, 用 于生成关键的季鏻盐中间体，从而实现 C-P 键的活化.

在以上这些反应中，作者认为反应是通过过渡金属 催化的方法，在反应过程中原位生成季鏻盐关键中间 体, 活化了 $\mathrm{C}-\mathrm{P}$ 键, 从而顺利实现 $\mathrm{C}-\mathrm{P}$ 键的转化反应.

然而，还有一些特殊的 $\mathrm{C}-\mathrm{P}$ 键活化反应，作者提 出了非季鏻盐的新的机制. 虽然这些反应机制可以解释 产物的生成, 但是难以阐释清楚反应高活性的来源, 特 别是 $\mathrm{C}-\mathrm{P}$ 键活化的驱动力. 相反, 利用类似的季鏻盐 中间体途径依然可以解释这些反应现象. 为此, 我们将 这些反应放在此部分进行介绍. 


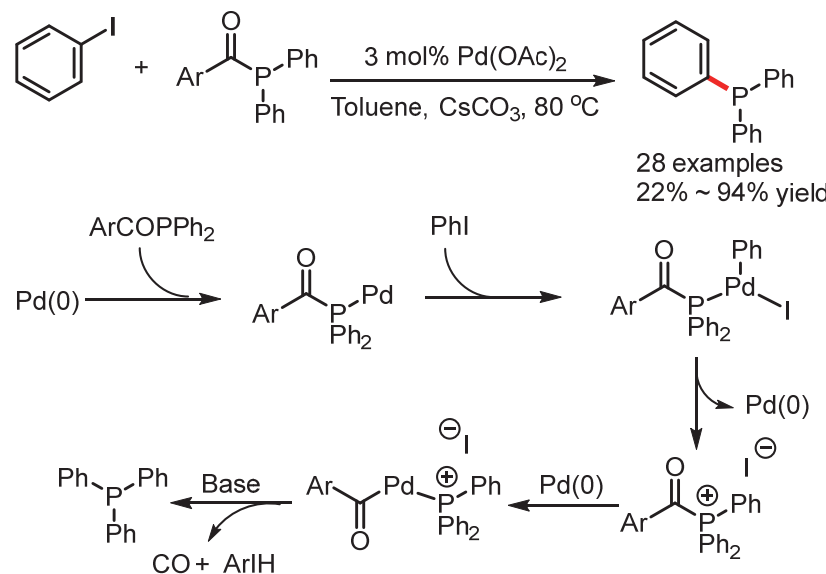

图 18 钯催化酰基膦和 PhI 的膦化反应

Figure 18 Pd-catalyzed phosophination of acylphosphine with $\mathrm{PhI}$
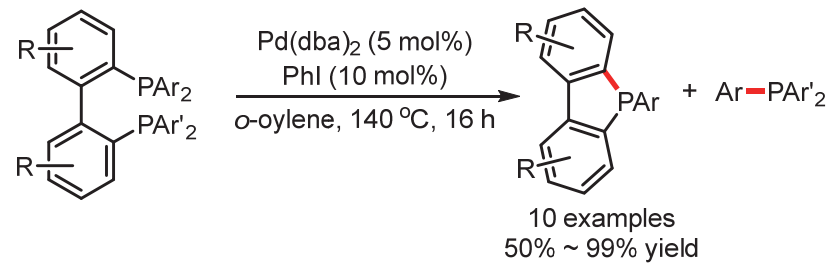

图 19 钯催化分子内膦化反应

Figure 19 Pd-catalyzed intramolecular phosphination

2013 年, $\mathrm{Wu}$ 课题组 ${ }^{[33]}$ 报道了廉价金属 $\mathrm{Ni}$ 催化的 $\mathrm{C}$ - $\mathrm{P}$ 活化反应(图 20). 反应以双芳基环丙萗烯烃和三 芳基膦为底物, $\mathrm{NiCl}_{2} \cdot 6 \mathrm{H}_{2} \mathrm{O}$ 为催化剂, $\mathrm{Zn}$ 为还原剂, 1,4二氧六环为溶剂, 能够以良好至优异的收率得到一系列 大位阻䒺基取代的芳基膦化合物. 作者提出的可能的机 理为: $\mathrm{Zn}$ 将 $\mathrm{Ni}\left(\mathrm{PPh}_{3}\right)_{2} \mathrm{Cl}_{2}$ 还原生成 $\mathrm{Ni}\left(\mathrm{PPh}_{3}\right)_{2}$, 随后环丙
烷 $\mathrm{C}-\mathrm{C}$ 键对 $\mathrm{Ni}\left(\mathrm{PPh}_{3}\right)_{2}$ 氧化加成生成金属环丁烷，该中 间体的 $\mathrm{Ni}-\mathrm{C}$ 与三苯基膦 $\mathrm{P}-\mathrm{C}$ 之间经由复分解实现 $\mathrm{C}-\mathrm{P}$ 键的活化, 苯基从 $\mathrm{P}$ 迁移到 $\mathrm{Ni}$ 并形成萗基 $\mathrm{C}-\mathrm{P}$ 键, 最后经还原消除得到产物. 该反应主要适用对称的 二芳基烯烃，当芳基取代基不一致时，反应的选择性较 差. 尽管这个反应是为数不多的 $\mathrm{Ni}$ 催化的 $\mathrm{C}-\mathrm{P}$ 活化例 子, 但是作者提出的复分解机制仍然不能较好解释二价 $\mathrm{Ni}$ 环中间体如何高效活化较为惰性的 $\mathrm{C}-\mathrm{P}$ 键. 因此, 通过季鏻盐中间体进行 $\mathrm{C}-\mathrm{P}$ 活化似乎也是一个不能被 排除的途径：四元 $\mathrm{Ni}$ 环与 $\mathrm{PPh}_{3}$ 配体还原消除得到季鏻 盐 $^{[34]}$, 随后 $\mathrm{Ni}$ 再与 $\mathrm{P}-\mathrm{Ph}$ 键氧化加成, 还原消除得到目 标产物. 由于季鏻盐的生成, $\mathrm{P}-\mathrm{Ph}$ 与 $\mathrm{Ni}$ 的氧化加成较 为容易，从而可以获得较高的反应活性.

2015 年, Mathey 和段征等 ${ }^{[35]}$ 实现了钯催化的炔基 导向 $\mathrm{C}-\mathrm{P}$ 活化、对炔烃的分子内加成反应(图 21). 反 应以 $10 \mathrm{~mol} \% \mathrm{Pd}(\mathrm{OAc})_{2}$ 和 $10 \mathrm{~mol} \% \mathrm{CuI}$ 为催化剂，甲苯 为溶剂, 在 $120{ }^{\circ} \mathrm{C}$ 下通过钯催化炔基取代的三芳基膦 底物 $\mathrm{C}-\mathrm{P}$ 键的活化, 合成了一系列五元膦环化合物, 获得中等至良好的收率.

作者推测反应的机理如下：P 原子与金属钯配位, 而后炔基作为扮演导向基团促进金属 $\mathrm{Pd}$ 与芳基 $\mathrm{C}-\mathrm{P}$ 键的氧化加成. 此外, 作者对相应的分子间反应也进行 了尝试，但并未得到理想的产物. 同上述例子一样，作 者给出的机理依然不能很好地解释炔烃导向作用如何 高效促进 $\mathrm{Pd}$ 与惰性 $\mathrm{C}-\mathrm{P}$ 键的氧化加成. 因此, 通过季 鏻盐中间体进行 $\mathrm{C}-\mathrm{P}$ 活化也是一个不能被排除的途径: $\mathrm{Cu}$ 盐活化炔烃, 然后分子内环化生成季鏻盐, $\operatorname{Pd}(0)$ 与 $\mathrm{P}$ - $\mathrm{Ph}$ 氧化加成, 再和烯基 $\mathrm{Cu}$ 转金属, 得到同样的 $\mathrm{Pd}$
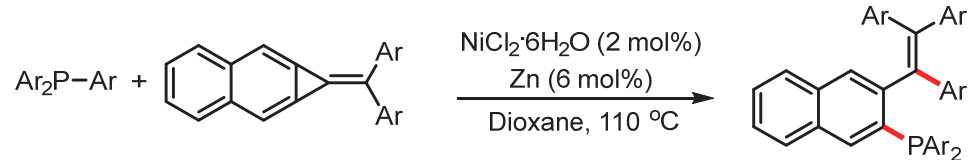

18 examples, $85 \% \sim 98 \%$ yield

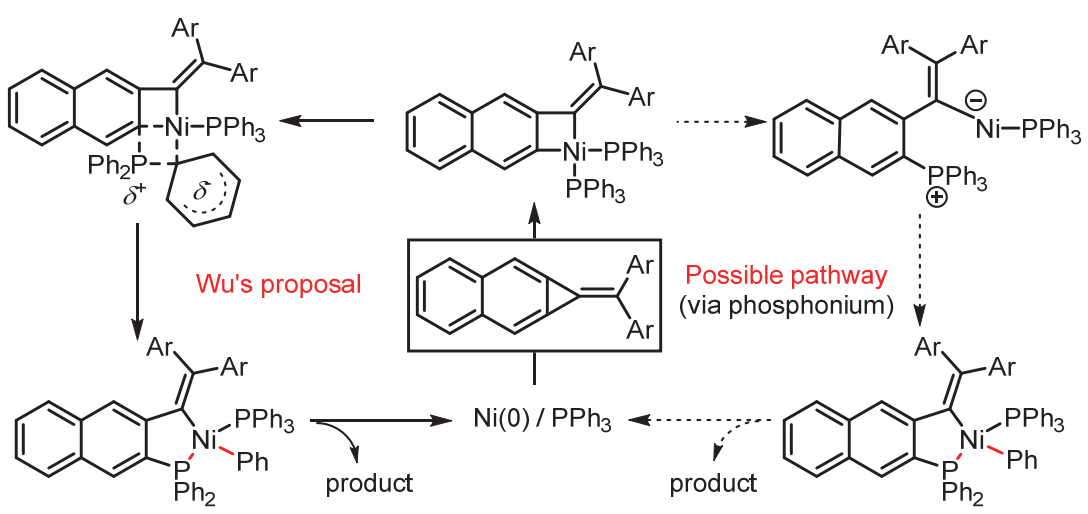

图 20 镍催化的分子分子间膦化反应

Figure 20 Ni-catalyzed intermolecular phosphination 
<smiles></smiles>

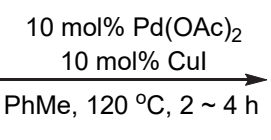<smiles>[Al]c1c(-c2ccccc2)c2ccccc2p1-c1ccccc1</smiles>

8 examples $29 \% \sim 72 \%$ yield
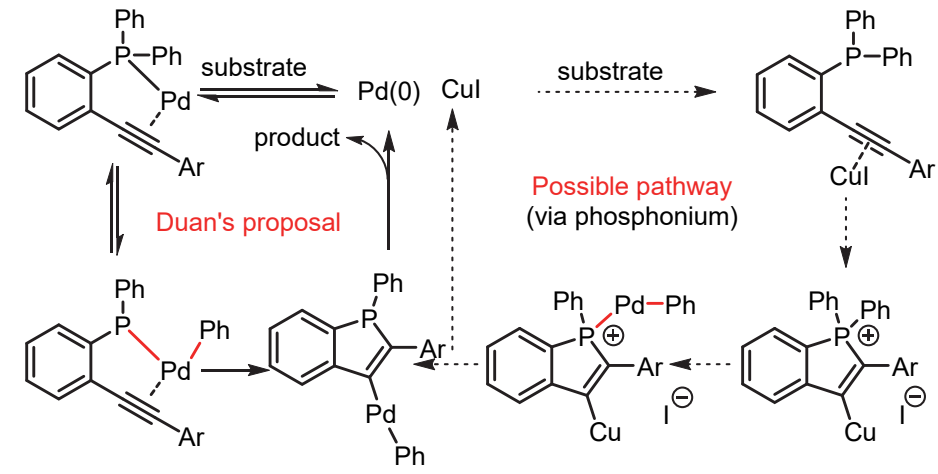

图 21 钯催化炔烃分子内芳膦化反应

Figure 21 Pd-catalyzed intramolecular arylphosphination

中间体，还原消除给出最终的产物。

2017 年，吴䂞等 ${ }^{[36]}$ 报道了吡唑作为螯合辅助基团 促进的 $\mathrm{Pd}$ 催化 $\mathrm{C}(\mathrm{O})-\mathrm{P}$ 键活化(图 22). 作者推测 $N$-溴 代丁二酰亚胺(NBS) 将 $\operatorname{Pd}(0)$ 氧化为 $\mathrm{Pd}(\mathrm{II})$, 继而 $\mathrm{C}$ $\mathrm{P}(\mathrm{O})$ 将 $\mathrm{Pd}(\mathrm{II})$ 氧化得到 $\mathrm{Pd}(\mathrm{IV})$ 络合物, 随后二苯基膦酸 阴离子亲核进攻丙二烯基团, 得到最终产物. 但是该机 理中, 弱的 $\mathrm{C}-\mathrm{P}$ 键与 $\mathrm{Pd}(\mathrm{II})$ 氧化加成得到 $\mathrm{Pd}(\mathrm{IV})$ 络合物 依然存有一定的争议. 因此, 通过季鏻盐中间体进行 $\mathrm{C}-\mathrm{P}$ 活化也是一个不能被排除的途径: 二价 $\mathrm{Pd}$ 物种和 $\mathrm{P}=\mathrm{O}$ 结合得到季鏻盐 ${ }^{[37]}$, 然后 $\mathrm{Pd}(0)$ 与 $\mathrm{C}-\mathrm{P}$ 键氧化加 成, 再通过基团迁移得到同样的中间体, 最后生成目标 产物.

\section{2 酰基膦促进 C-P 键活化}

通过形成季鏻盐的方式可以增强 $\mathrm{P}$ 端的缺电性, 从
而减弱 $\mathrm{C}-\mathrm{P}$ 键的键能, 相应地, 通过增加 $\mathrm{C}$ 端的缺电 性, 也可以促进 C-P 键对金属的氧化加成. 1989 年, Nakazawa 等[38]报道了钯催化酰基磷酸酯的脱羰反应(图 23). 作者发现 $\mathrm{Pd}($ styrene $)\left(\mathrm{PMePh}_{2}\right)_{2} 、 \operatorname{Pd}\left(\mathrm{PPh}_{3}\right)_{4}$ 、 $\operatorname{Pd}\left(\mathrm{PCy}_{3}\right)_{2}$ 等 $\mathrm{Pd}(0)$ 前体均具有一定的催化活性, 而不能 形成 $\mathrm{Pd}(0)$ 物种的 $\mathrm{PdCl}_{2}\left(\mathrm{PPh}_{3}\right)_{2} 、 \mathrm{Pd}(\mathrm{acac})_{2}$ 等则不能催化 反应. 此外, 芳基酰基磷酸酯表现出比烷基酰基磷酸酯 更高的反应活性. 作者推测反应经历了 $\mathrm{C}-\mathrm{P}$ 键与低价 钯氧化加成的关键步骤.

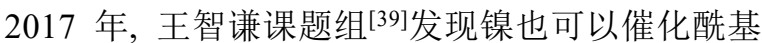
$\mathrm{C}-\mathrm{P}$ 键的活化(图 24). 以酰基膦为底物，5 $\mathrm{mol} \%$ $\mathrm{NiCl}_{2}$ (dppp)为金属催化剂, 二甲苯为溶剂, $150{ }^{\circ} \mathrm{C}$ 条件 下可以得到脱羰基的三取代膦化合物. 作者提出的机理 为, $\mathrm{C}(\mathrm{O})-\mathrm{P}$ 键与金属镍氧化加成, 经脱羰基、还原消<smiles>[R]/C(=C/C(C(=O)P)=C(/[R])Cn1nc([R3])cc1[R])C(=O)O</smiles><smiles>C[PbH2]</smiles>

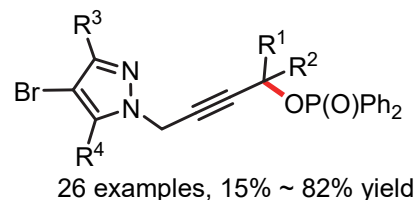

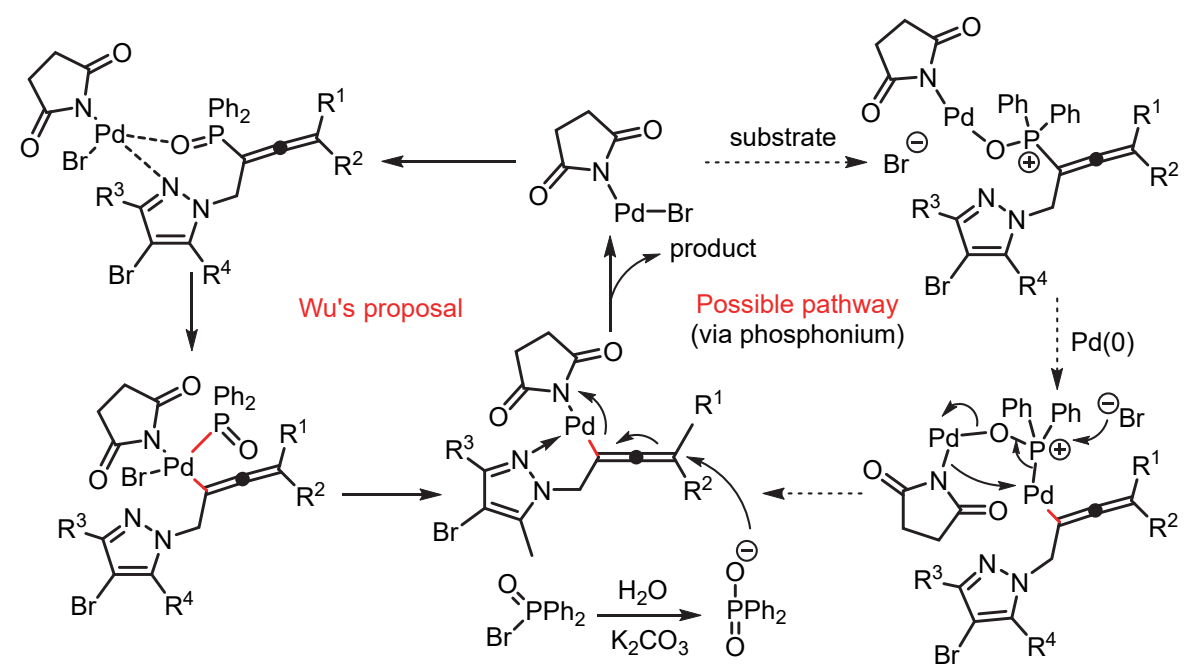

图 22 吡唑导向的 C-P 键活化转化

Figure $22 \mathrm{C}-\mathrm{P}$ bond activation via pyrazole direction 


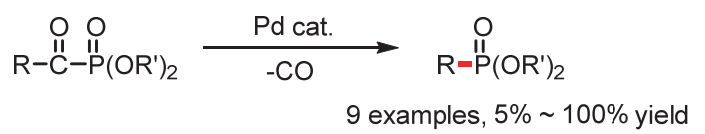

图 23 钯催化脱羰型 $\mathrm{C}-\mathrm{P}$ 键活化转化

Figure 23 Pd-catalyzed decarbonylation-type $\mathrm{C}-\mathrm{P}$ transformation

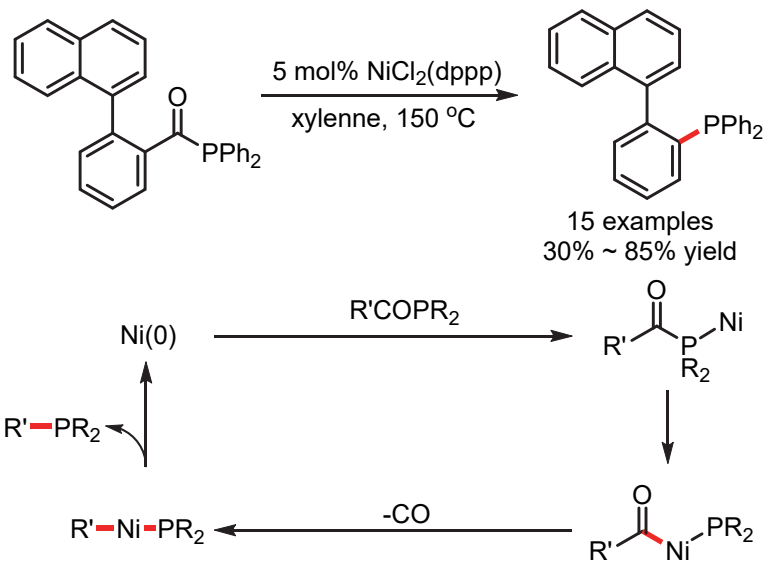

图 24 镍催化脱羰型 $\mathrm{C}-\mathrm{P}$ 键活化转化

Figure 24 Ni-catalyzed decarbonylation-type $\mathrm{C}-\mathrm{P}$ transformation

除得到最终产物.

作为进一步的拓展, 2021 年, 王智谦课题组 ${ }^{[40]}$ 还尝 试了金属钯催化芳酰基膦氧化物的 $\mathrm{C}-\mathrm{P}(\mathrm{V})$ 键活化(图 $25)$. 反应以 $1 \mathrm{~mol} \% \mathrm{Pd}_{2}(\mathrm{dba})_{3}$ 为金属催化剂, 以 $8 \mathrm{~mol} \%$ $\mathrm{PCy}_{3}$ 为配体, 以二甲苯为溶剂, 在 $130{ }^{\circ} \mathrm{C}$ 条件下可以得 到一系列脱羰三芳基膦氧产物.

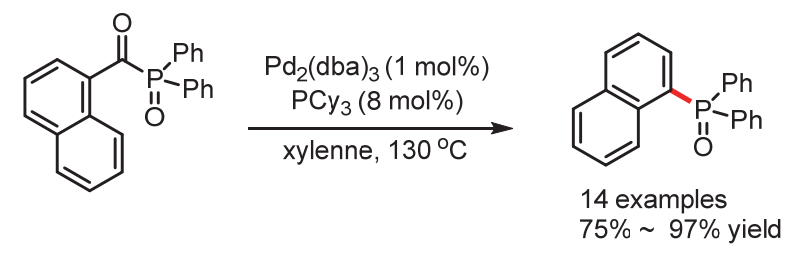

图 25 钯催化脱羰型 $\mathrm{C}-\mathrm{P}$ 键活化转化

Figure 25 Pd-catalyzed decarbonylation-type $\mathrm{C}-\mathrm{P}$ transformation

\section{3 环张力促进 C-P 键活化}

除了通过增强底物 $\mathrm{C}-\mathrm{P}$ 键的缺电性来促进氧化加 成的方法外, 引入较大的张力环也可以促进 $\mathrm{C}-\mathrm{P}$ 键的 氧化加成.

1999 年, Grützmacher 等[41]发现多环骨架膦化合物 的三元环 $\mathrm{C}-\mathrm{P}$ 键可以与 $[\mathrm{Rh}(\operatorname{cod}) \mathrm{Cl}]_{2}$ 进行氧化加成, 释 放环张力, 从而实现 $\mathrm{C}-\mathrm{P}$ 键的活化, 但是该工作未实 现金属的催化循环(图 26).

\section{4 导向基促进 C-P 键活化}

以上策略均是通过增强缺电性或环张力来减弱 $\mathrm{C}$ -

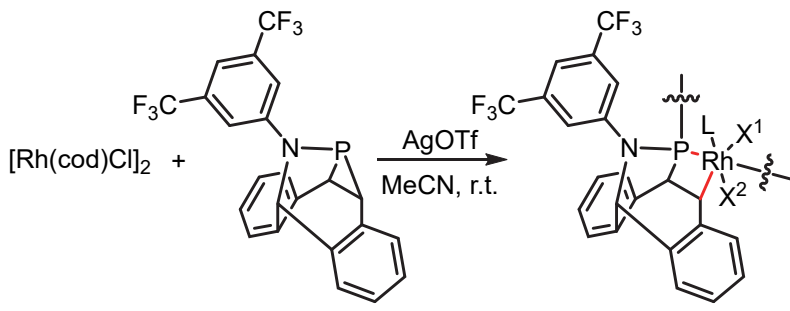

图 26 张力环促进的 $\mathrm{C}-\mathrm{P}$ 键活化转化

Figure 26 Strained ring-enabled $\mathrm{C}-\mathrm{P}$ transformation

$\mathrm{P}$ 键共价键的强度, 从而促进 $\mathrm{C}-\mathrm{P}$ 键和金属的氧化加 成. 与此不同的是，利用分子内的配位基团与金属的配 位可以拉近金属和 $\mathrm{C}-\mathrm{P}$ 键的距离, 进而发生类似分子 内的反应，也可以促进 $\mathrm{C}-\mathrm{P}$ 键的活化. 然而这类反应 活性依然不高, 多数反应难以实现金属的催化循环.

2002 年, Heyn 等[42]报道 $\mathrm{Pd}_{2}(\mathrm{dba})_{3}$ 介导的五氟苯基 取代的双膦化合物的 $\mathrm{C}-\mathrm{P}$ 键断裂, 生成钯二聚体(图 27). 其中一个 $\mathrm{P}$ 原子作为配位性导向基团与 $\mathrm{Pd}$ 配位, 从而促进对另一个芳基膦的 $\mathrm{C}-\mathrm{P}$ 键活化. 同时, 五氟 苯基作为强吸电子基团降低了 $\mathrm{C}-\mathrm{P}$ 键的电子密度，进 一步促进了 $\mathrm{C}-\mathrm{P}$ 键和低价过渡金属的氧化加成. 当使 用其他取代基代替 $\mathrm{C}_{6} \mathrm{~F}_{5}$ 基团时，反应将难以发生.
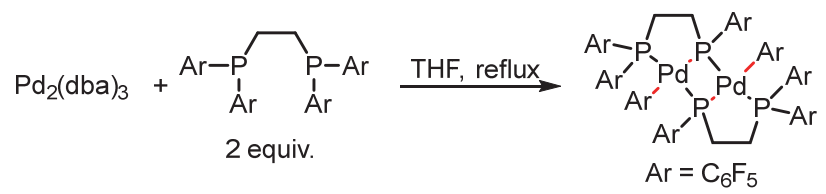

图 $27 \mathrm{P}$ 原子导向的 $\mathrm{C}-\mathrm{P}$ 键活化

Figure $27 \mathrm{P}$ atom-directed $\mathrm{C}-\mathrm{P}$ bond activation

2011 年, Bourissou 等 ${ }^{[43]}$ 报道了含双 $\mathrm{P}$ 取代基的 $\mathrm{P}(\mathrm{O})$ 底物的 $\mathrm{C}-\mathrm{P}(\mathrm{V})$ 键活化(图 28). 底物的两个三价 $\mathrm{P}$ 原子 与 $\mathrm{Pd}$ 配位, 拉近金属与 $\mathrm{P}(\mathrm{O})-\mathrm{Ph}$ 的距离, 从而实现了 $\mathrm{C}-\mathrm{P}(\mathrm{V})$ 键的活化.

导向基团辅助的 $\mathrm{C}-\mathrm{P}$ 键活化反应的最大难点在于 如何获得金属的催化循环，可能的原因在于导向基和金 属有着较强的配位，导致金属难以解离，实现催化循环. 此外，含 $\mathrm{P}$ 导向基常常具有较大的位阻，也妨碍了反应 试剂和金属的接近，影响了反应的活性.

\section{5 催化剂促进 C一P 键活化}

形成季鏻盐、构建张力环、引入吸电子取代基和安 装导向基等需底物预活化方法可以较好地实现 $\mathrm{C}-\mathrm{P}$ 键 活化, 但无需底物预活化是实现 $\mathrm{C}-\mathrm{P}$ 键的直接活化转 化最为经济和高效的方法. 但是由于 $\mathrm{C}-\mathrm{P}$ 键的特殊性, 此方向的发展非常缓慢.

实际上，早在 1984 年, Bryant 等 ${ }^{[4]}$ 在研究丙烯氢酰 化的副反应时，发现在铑催化剂作用下不同三芳基膦化 合物之间的芳基交换现象(图 29). 不同于此前发现的等 

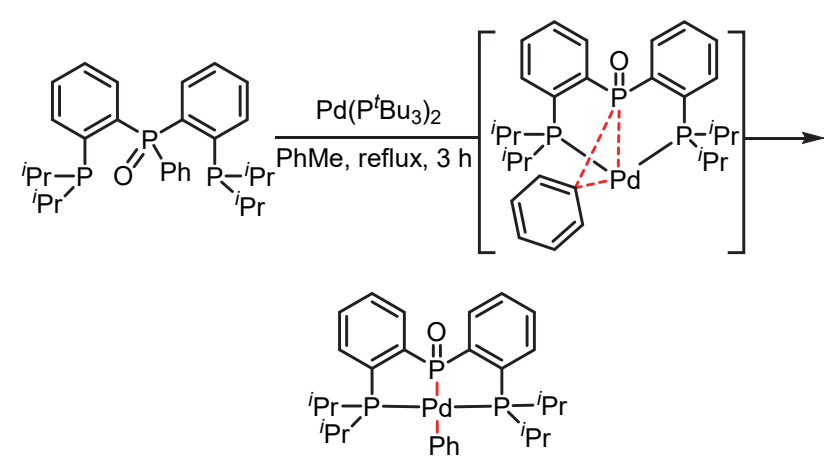

图 28 双齿导向基促进的 $\mathrm{C}-\mathrm{P}$ 键活化

Figure 28 Bidentate group-enabled $\mathrm{C}-\mathrm{P}$ bond activation

物质的量的金属和膦的配合物之间的芳基交换，该反应 中所用膦化合物的物质的量为铑催化剂的 20 倍, 暗示 该反应有望实现 $\mathrm{C}-\mathrm{P}$ 键的催化活化反应. 作者发现以 $\left[\mathrm{Rh}\left(\mathrm{PPh}_{3}\right)_{3}(\mathrm{H})(\mathrm{CO})\right]$ 为催化剂, 该膦的芳基交换反应速 率受温度的影响较为明显. 通过将反应温度提升至 $170{ }^{\circ} \mathrm{C}$, 可以使得 $130{ }^{\circ} \mathrm{C}$ 条件下已经失活的体系重新 具备反应活性. 此外, 反应还受 $\mathrm{Rh}$ 催化剂前体结构的 影响, 包括 $\mathrm{Rh}(\mathrm{acac})(\mathrm{CO}) \mathrm{Ph}_{3} \mathrm{P}$ 和 $\mathrm{RhCl}\left(\mathrm{Ph}_{3} \mathrm{P}\right)_{3}$ 在内的单 核配合物表现出较高的催化活性, 而 $\mathrm{Rh}_{6}(\mathrm{CO})_{16}$ 等多配 位的、结构稳定的金属簇催化的反应速率则较低. 由此, 作者推测, 膦化合物的 $\mathrm{C}-\mathrm{P}$ 键对具有更高催化活性的 单核 $\mathrm{Rh}$ 物种进行氧化加成, 得到中间体 $\mathbf{A}$ 和 $\mathbf{B}$, 并形成 膦桥二聚体 $\mathbf{C}$, 基于该中间体的配体交换和随后的还原 消除, 实现了该类芳基交换催化循环. 值得一提的是, 除了 $\mathrm{Rh}$ 之外的其他过渡金属催化剂, 如 $\mathrm{Co}_{2}(\mathrm{CO})_{8}$ 、 $\mathrm{Os}_{3}(\mathrm{CO})_{12} 、 \mathrm{Ni}(\mathrm{CO})_{2}\left(\mathrm{Ph}_{3} \mathrm{P}\right)_{2} 、 \mathrm{Pd}\left(\mathrm{PPh}_{3}\right)_{4} 、 \mathrm{Ru}_{3}(\mathrm{CO})_{12}$ 、 $\mathrm{Os}_{3}(\mathrm{CO})_{12}$ 等也具有一定的催化活性. 然而, 自此以后, 这个发现一直没有后续的跟进和进一步的突破.

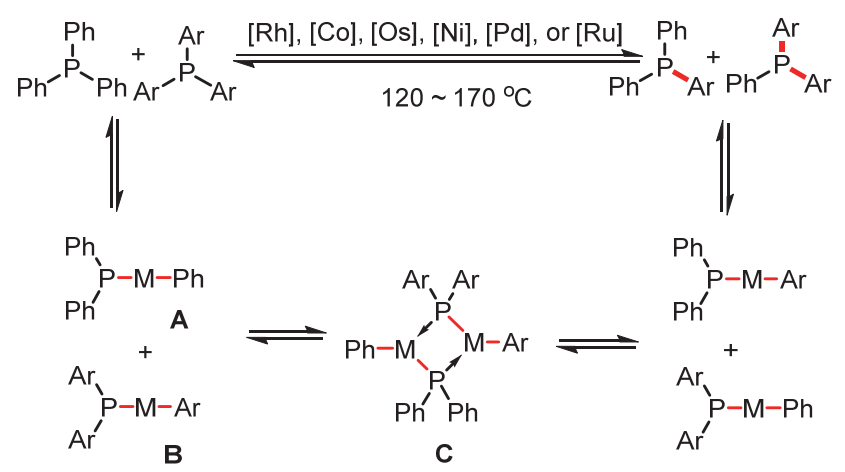

图 29 催化剂促进的 $\mathrm{C}-\mathrm{P}$ 键的活化

Figure 29 Catalyst-enabled $\mathrm{C}-\mathrm{P}$ bond activation

2017 年, Whittlesey 等 ${ }^{[45]}$ 研究 $\mathrm{Ni}(\mathrm{cod})_{2}$ 与 6-Mes 和 $\mathrm{PPh}_{3}$ 的 $1: 2$ (物质的量比)混合物反应时, 发现生成了三 配位 $\mathrm{Ni}(0)$ 氮杂环卡宾配合物 [Ni(6-Mes) $\left.\left(\mathrm{PPh}_{3}\right)_{2}\right]$ (6,6-Mes $=1,3$-双 (2,4,6-三甲基苯基)-3,4,5,6-四氢嘧啶-2-
亚基)(图 30). 作者推测在五元环 $\mathrm{N}$-杂环卡宾 $\mathrm{IMe}_{4}$ 作用 下, 可以发生 $\mathrm{PPh}_{3}$ 与 $\mathrm{Ni}$ 的氧化加成, 得到络合物 $\left[\mathrm{Ni}\left(\mathrm{IMe}_{4}\right)_{2}\left(\mathrm{PPh}_{2}\right) \mathrm{Ph}\right]$. 然而该反应是在等物质的量的镍 络合物存在下进行的, 还无法实现金属的催化循环.<smiles>CN1CCCN(C)C1N(P)c1ccccc1</smiles><smiles>CCCCCCC(C)C</smiles>

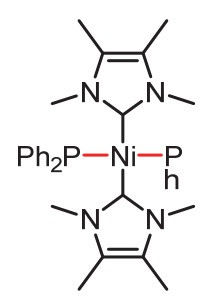

$67 \%$ yield
图 30 NHC-Ni 催化剂促进的 C-P 键的活化

Figure 30 NHC-Ni-enabled $\mathrm{C}-\mathrm{P}$ bond activation

\section{2 通过过渡金属诱导的极性键的加成消去途径 实现 C-P 键活化}

尽管大部分的 $\mathrm{C}-\mathrm{P}$ 活化通过 $\mathrm{C}-\mathrm{P}$ 键与低价金属 的氧化加成机制进行, 但是当高价膦存在时, 可以发生 类似羰基化合物的加成消去机理进行 $\mathrm{C}-\mathrm{P}$ 的断裂. 不 过此处的高价金属更多的是充当 Lewis 酸诱导作用，没 有直接参与 $\mathrm{C}-\mathrm{P}$ 的断裂. 考虑到离解生成的芳基取代 基可以和金属结合, 参与各类过渡金属催化的偶联反 应，我们对这类反应也进行了介绍.

2003 年, Shinokubo 和 Oshima 等 ${ }^{[46]}$ 报道了芳基磷酸 作为芳基化试剂与烯烃的 Heck 烯基化反应(图 31). 其 中, 四正丁基氟化铵(TBAF)对反应有关键的作用. 鉴于 $\mathrm{F}$ 和 $\mathrm{P}$ 之间较强的亲和性，作者提出 $\mathrm{F}^{-}$进攻芳基磷酸形 成五配位的磷中间体，该中间体的 $\mathrm{O}$ 原子与 $\mathrm{Pd}(\mathrm{II})$ 配位, 进而发生 $\mathrm{Pd}-\mathrm{O}$ 与 $\mathrm{C}-\mathrm{P}$ 之间的复分解, 实现 $\mathrm{C}-\mathrm{P}$ 活化. 而氧化剂 $\mathrm{Me}_{3} \mathrm{NO} \cdot 2 \mathrm{H}_{2} \mathrm{O}$ 可以将 $\mathrm{Pd}(0)$ 氧化为具有反应活 性的 $\mathrm{Pd}(\mathrm{II})$ 物种. 所形成的磷酸 $\mathrm{H}_{3} \mathrm{PO}_{4}$ 副产物通过 ${ }^{31} \mathrm{P}$ NMR 得到了确认.

2011 年, Tanaka 课题组 ${ }^{[47]}$ 报道了铑催化的炔酫与酰 基磷酸酯的环化反应(图 32). 作者推测醛羰基和炔基对 $\mathrm{Rh}(\mathrm{I})$ 进行氧化环加成后, 存在两种可能的反应途径: 经 过 $\mathrm{Rh}-\mathrm{O}$ 对 $\mathrm{P}=\mathrm{O}$ 键的加成消除途径实现 $\mathrm{C}-\mathrm{P}$ 转化 (path 1), 或者经过 $\mathrm{Rhh}-\mathrm{O}$ 与 $\mathrm{C}(\mathrm{O})-\mathrm{P}$ 发生复分解实现 $\mathrm{C}-\mathrm{P}$ 转化 (path 2). 这是目前唯一一例在 $\mathrm{C}-\mathrm{P}$ 活化反应 中存在不对称控制的例子，但是考虑到手性中心的产生 和 $\mathrm{C}-\mathrm{P}$ 转化没有直接的关系, 不对称 $\mathrm{C}-\mathrm{P}$ 活化转化依 然是一个没有解决的挑战.

\section{3 总结和展望}

过渡金属催化的 $\mathrm{C}-\mathrm{P}$ 键活化转化可以使用多样性 的有机膦化物作为合成砌块, 为各类有机膦化合物的高 


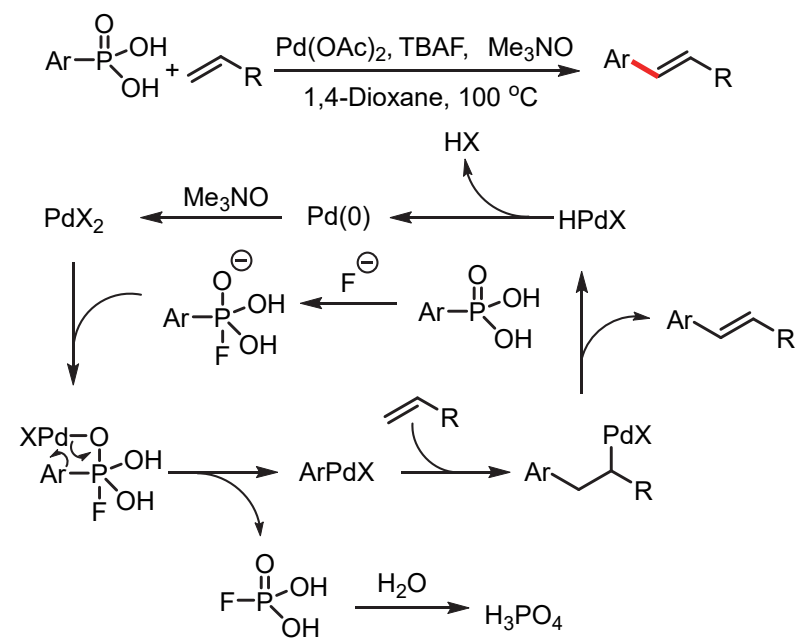

图 31 通过 $\mathrm{P}=\mathrm{O}$ 加成消去途径实现的 $\mathrm{C}-\mathrm{P}$ 键的转化

Figure 31 Transformation of $\mathrm{C}-\mathrm{P}$ bond by addition-elimination pathway of $\mathrm{P}=\mathrm{O}$ bond
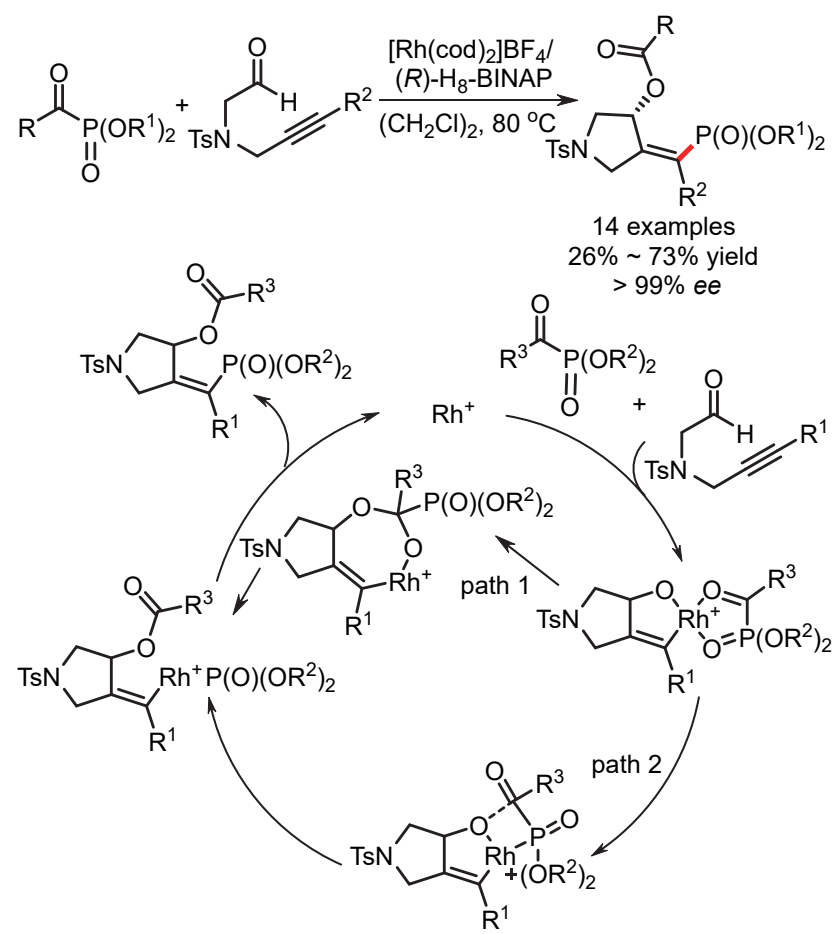

图 32 通过 $\mathrm{P}-\mathrm{C}(\mathrm{O})$ 加成消去途径实现的 $\mathrm{C}-\mathrm{P}$ 键的转化

Figure 32 Transformation of $\mathrm{C}-\mathrm{P}$ bond by addition-elimination pathway of $\mathrm{P}-\mathrm{C}(\mathrm{O})$ bond

效构建提供了一条原子和步骤经济的途径，因而受到了 越来越多的关注. 然而由于 $\mathrm{C}-\mathrm{P}$ 键较高的键能以及 $\mathrm{P}$ 原子具有较强的配位能力, 过渡金属催化的 $\mathrm{C}-\mathrm{P}$ 键活 化一直是个较大的挑战. 目前通过底物的活化方法, 包 括预先或者在反应过程中原位生成季鏻盐的方法、引入 酰基膦或者膦氧、构建张力环以及安装特殊导向基的方 法, 可以促进 $\mathrm{C}-\mathrm{P}$ 的活化转化. 其中, 仅有季鏻盐的方 法发展相对成熟, 不仅反应活性高, 而且反应类型也相
对多样．但是依然存在明显的局限性：预先生成季鏻盐 的方法往往需要额外反应步骤, 且原料的纯化也较为困 难; 现场生成季鏻盐的方法活性依然有限，多数局限于 分子内反应，常常还需要导向基的辅助. 相比之下，其 他类型方法的发展更为有限，多数还不能实现金属的催 化循环. 特别是导向基辅助的方法, 尽管在 $\mathrm{C}-\mathrm{H} 、 \mathrm{C}-$ $\mathrm{C} 、 \mathrm{C}-\mathrm{N}$ 等惰性的活化中取得了很大成功，但是在 $\mathrm{C}-$ $\mathrm{P}$ 键的活化中还没有取得重要突破，尤其是实现金属的 催化循环. 相比于底物预活化的方法, 不需要底物活化 的直接 $\mathrm{C}-\mathrm{P}$ 的活化转化是最为吸引人的方法, 但是从 最早的一篇报道之后，几乎没有后续的跟进研究，暗示 这个方法还面临着相当大的挑战，亟待广泛而深入的研 究.

除了各类活化策略外，底物类型和金属类型也存在 很多的问题. 目前所报道的反应大多集中于 $\mathrm{C}\left(\mathrm{sp}^{2}\right)-\mathrm{P}$ 键活化, 而更加惰性的 $\mathrm{C}\left(\mathrm{sp}^{3}\right)-\mathrm{P}$ 键活化则仍然较少, 特别是在季鏻盐的基团交换反应中，芳基基团比烷基基 团更易于交换. 因此，针对烷基类基团，需要发展更加 高效的催化体系. 此外, 过渡金属催化剂仍然以贵金属 为主，廉价金属仍然面临活性低，反应类型和底物类型 局限的问题.

此外，由于反应活性仍然不高，手性配体选择有限， 对映选择性控制的 $\mathrm{C}-\mathrm{P}$ 键活化转化一直是一个很大的 挑战，到目前为止还没有成功的例子.

总之, 过渡金属催化的 $\mathrm{C}-\mathrm{P}$ 键活化在近些年受到 越来越多的关注并已取得明显的进展，为有机膦化合物 的应用和合成提供了一种更为经济、高效的方法，但是 未来还需要更多的努力来进一步提高 $\mathrm{C}-\mathrm{P}$ 键活化转化 反应的活性和选择性，推动该类型反应在合成中的广泛 应用。

\section{References}

[1] (a) Fields, S. C. Tetrahedron 1999, 55, 12237.

(b) Engel, R. Chem. Rev. 1977, 77, 349.

[2] (a) Wittig, G.; Geissler, G. Justus Liebigs Ann. Chem. 1953, 580, 44.

(b) Lu, X. Y.; Zhang, C. M.; Xu, Z. R. Acc. Chem. Res. 2001, 34, 535 .

[3] (a) Trofimov, B. A.; Arbuzova, S. N.; Gusarova, N. K. Russ. Chem. Rev. 1999, 68, 215

(b) Honaker, M. T.; Hovland, J. M.; Salvatore, R. N. Curr. Org. Chem. 2007, 4, 31 .

(c) Schwan, A. L. Chem. Soc. Rev. 2004, 33, 218.

[4] (a) Jiang, T.; Zhang, H.; Ding, Y.; Zou, S.; Chang, R.; Huang, H. Chem. Soc. Rev. 2020, 49, 1487.

(b) Lee, Y. H.; Morandi, B. Coord. Chem. Rev. 2019, 386, 96.

(c) Wang, L.; Chen, H.; Duan, Z. Chem. Asian J. 2018, 13, 2164.

(d) Tappe, F. M. J.; Trepohl, V. T.; Oestreich, M. Synthesis 2010, 3037.

(e) Macgregor, S. A. Chem. Soc. Rev. 2007, 36, 67.

[5] Wei, K.; Luo, K.; Liu, F.; Wu, L.; Wu, L.-Z. Org. Lett. 2019, 21 , 1994. 
[6] (a) Liu, T.; Zhu, J.; Sun, X.; Cheng, L.; Wu, L. Adv. Synth. Catal. 2019, 361, 3532.

(b) Hou, F.; Du, X.-P.; Alduma, A. I.; Li, Z.-F.; Huo, C.-D. Wang, X.-C.; Wu, X.-F.; Quan, Z.-J. Adv. Synth. Catal. 2020, 362, 4755.

(c) Song, Y.; Wang, L.; Duan, Z.; Mathey, F. Chin. Chem. Lett. 2020, 31, 329 .

[7] For selected reviews on $\mathrm{C}-\mathrm{H}$ activation, see:

(a) Gandeepan, P.; Müller, T.; Zell, D.; Cera, G.; Warratz, S.; Ackermann, L. Chem. Rev. 2019, 119, 2192.

(b) Zhang, Q.; Shi, B. F. Acc. Chem. Res. 2021, 54, 2750.

(c) He, J.; Wasa, M.; Chan, Kelvin S. L.; Shao, Q.; Yu, J. Q. Chem. Rev. 2017, 117, 8754.

(d) Davison, R. T.; Kuker, E. L.; Dong, V. M. Acc. Chem. Res. 2021, 54, 1067.

(e) Ye, B.; Cramer, N. Acc. Chem. Res. 2015, 48, 1308.

(f) Hummel, J. R.; Boerth, J. A.; Ellman, J. A. Chem. Rev. 2017, $117,9163$.

(g) Dong, Z.; Ren, Z.; Thompson, S.; Xu, Y.; Dong, G. B. Chem. Rev. 2017, 117, 9333.

(h) Hu, Y.; Wang, C. Acta Phys.-Chim. Sin. 2019, 35, 913.

(i) Wang, Y.-X.; Ye, M. Sci. China Chem. 2018, 61, 1004.

(j) Wang, R.; Luan, Y.; Ye, M. Chin. J. Chem. 2019, 37, 720.

(k) Li, R.; Xu, X.; Ye, M. Chin. J. Org. Chem. 2020, 40, 3196 (in Chinese).

(李然, 徐学涛, 叶萌春, 有机化学, 2020, 40, 3196.)

(1) Zhao, M.; Lu, W. Acta Phys.-Chim. Sin. 2019, 35, 977.

[8] For selected reviews on $\mathrm{C}-\mathrm{C}$ activation, see:

(a) Xia, Y.; Dong, G. Nat. Rev. Chem. 2020, 4, 600.

(b) Souillart, L.; Cramer, N. Chem. Rev. 2015, 115, 9410.

(c) Song, F. J.; Gou, T.; Wang, B. Q.; Shi, Z. J. Chem. Soc. Rev. 2018, 47, 7078 .

(d) Murakami, M.; Ishida, N. Chem. Rev. 2021, 121, 264.

(e) Wang, W.; Xie, J. Chin. J. Org. Chem. 2020, 40, 1396 (in Chinese).

(王文亮，谢劲，有机化学, 2020, 40, 1396.)

[9] For selected reviews on $\mathrm{C}-\mathrm{O}$ activation, see:

(a) Liu, F.; Jiang, H.J.; Zhou, Y.; Shi, Z. J. Chin. J. Chem. 2020, 38, 855.

(b) Tobisu, M.; Chatani, N. Acc. Chem. Res. 2015, 48, 1717.

(c) Su, B.; Cao, Z.-C.; Shi, Z.-J. Acc. Chem. Res. 2015, 48, 886.

(d) Goossen, L. J.; Goossen, K.; Stanciu, C. Angew. Chem., Int. Ed. 2009, 48, 3569 .

[10] For selected reviews on $\mathrm{C}-\mathrm{N}$ activation, see:

(a) García-Cárceles, J.; Bahou, K. A.; Bower, J. F. ACS Catal. 2020, 10, 12738.

(b) Wang, Q. J.; Su, Y. J.; Li, L. X.; Huang, H. M. Chem. Soc. Rev. 2016, $45,1257$.

(c) Boit, T. B.; Bulger, A. S.; Dander, J. E.; Garg, N. K. ACS Catal. 2020, 10, 12109.

(d) Kaiser, D.; Bauer, A.; Lemmerer, M.; Maulide, N. Chem. Soc. Rev. 2018, 47, 7899.

(e) Gao, Y.; Ji, C.-L.; Hong, X. Sci. China Chem. 2017, 60, 1413.

[11] O'Keefe, D. F.; Dannock, M. C.; Marcuccio, S. M. Tetrahedron Lett. 1992, 33, 6679.

[12] Sakamoto, M.; Shimizu, I.; Yamamoto, A. Chem. Lett. 1995, 24, 1101.

[13] Brunker, T. J.; Moncarz, J. R.; Glueck, D. S.; Zakharov, L. N.; Golen, J. A.; Rheingold, A. L. Organometallics 2004, 23, 2228.

[14] Marinetti, A.; Carmichael, D. Chem. Rev. 2002, 102, 201.

[15] Landis, C. R.; Nelson, R. C; Jin, W.; Bowman, A. C. Organometallics 2006, 25, 1377.

[16] Rünzi, T.; Tritschler, U.; Roesle, P.; Göttker-Schnetmann, I.; Möller, H. M.; Caporaso, L.; Poater, A.; Cavallo, L.; Mecking, S. Organo- metallics 2012, 31, 8388 .

[17] Alcazar-Roman, L. M.; Hartwig, J. F.; Rheingold, A. L.; Liable-Sands, L. M.; Guzei, I. A. J. Am. Chem. Soc. 2000, 122, 4618.

[18] Ge, S. Z.; Green, R. A.; Hartwig, J. F. J. Am. Chem. Soc. 2014, 136, 1617.

[19] Ai, P. F.; Danopoulos, A. A.; Braunstein, P. Dalton Trans. 2014, 43, 1957.

[20] Qin, H.-L.; Leng, J.; Zhang, W.; Kantchev, E. A. B. Dalton Trans. 2018, 47, 2662.

[21] Vicente, J.; Abad, J.-A.; López-Nicolás, R.-M.; Jones, P. G. Organometallics 2004, 23, 4325.

[22] Hwang, L. K.; Na, Y.; Lee, J.; Do, Y.; Chang, S. Angew. Chem., Int. Ed. 2005, 44, 6166.

[23] Zhang, X.; McNally, A. Angew. Chem., Int. Ed. 2017, 56, 9833.

[24] Segelstein, B. E.; Butler, T. W.; Chenard, B. L. J. Org. Chem. 1995, 60,12 .

[25] Kong, K. C.; Cheng, C. H. J. Am. Chem. Soc. 1991, 113, 6313.

[26] Goodson, F. E.; Wallow, T. I.; Novak, B. M. J. Am. Chem. Soc. 1997, 119,12441.

[27] (a) Kwong, F. Y.; Chan, K. S. Chem. Commun. 2000, 1069. (b) Kwong, F. Y.; Lai, C. W.; Tian, Y.; Chan, K. S. Tetrahedron Lett. 2000, 41,10285.

(c) Kwong, F. Y.; Lai, C. W.; Yu, M.; Chan, K. S. Tetrahedron 2004, 60,5635 .

[28] Baba, K.; Tobisu, M.; Chatani, N. Angew. Chem., Int. Ed. 2013, 52, 11892.

[29] Baba, K.; Tobisu, M.; Chatani, N. Org. Lett. 2015, 17, 70.

[30] Zhou, H.; Li, J.; Yang, H.; Xia, C.; Jiang, G. Org. Lett. 2015, 17 , 4628.

[31] Yu, R.; Chen, X.; Wang, Z. Tetrahedron Lett. 2016, 57, 3404.

[32] Lian, Z.; Bhawal, B. N.; Yu, P.; Morandi, B. Science 2017, 356, 1059.

[33] Cao, J.; Huang, X.; Wu, L. Chem. Commun. 2013, 49, 7747.

[34] For nickel intermediates with negative charge, see: (a) Gartia, Y.; Ramidi, P.; Jones, D. E.; Pulla, S.; Ghosh, A. Catal. Lett. 2014, 144, 507.

(b) Sahoo, D.; Yoo, C.; Lee, Y. J. Am. Chem. Soc. 2018, 140, 2179.

[35] Zhou, Y.; Gan, Z. J.; Su, B.; Li, J.; Duan, Z.; Mathey, F. Org. Lett. 2015, 17, 5722 .

[36] Zhu, J.; Mao, M.; Ji, H.-J.; Xu, J.-Y.; Wu, L. Org. Lett. 2017, 19 , 1946.

[37] For the coordination of phosphine oxide with metals, see: (a) Veith, M.; Huch, V. J. Organomet. Chem. 1985, 293, 161. (b) Zabula, A. V.; Pape, T.; Hepp, A.; Hahn, F. E. Dalton Trans. 2008, 5886.

[38] Nakazawa, H.; Matsuoka, Y.; Yamaguchi, H.; Kuroiwa, T.; Miyoshi, K.; Yoneda, H. Organometallics 1989, 8, 2272.

[39] Yu, R.; Chen, X.; Martin, S. F.; Wang, Z. Org. Lett. 2017, 19, 1808.

[40] Chen, X.; Liu, X.; Zhu, H.; Wang, Z. Tetrahedron 2021, 81, 131912.

[41] Liedtke, J.; Rüegger, H.; Loss, S.; Grützmacher H. Angew. Chem., Int. Ed. 2000, 39, 2478.

[42] Heyn, R. H.; Görbitz, C. H. Organometallics 2002, 21, 2781.

[43] Derrah, E. J.; Ladeira, S.; Bouhadir, G.; Miqueu, K.; Bourissou, D. Chem. Commun. 2011, 47, 8611.

[44] Abatjoglou, A. G.; Bryant, D. R. Organometallics 1984, 3, 932.

[45] Sabater, S.; Page, M. J.; Mahon, M. F.; Whittlesey, M. K. Organometallics 2017, 36, 1776.

[46] Inoue, A.; Shinokubo, H.; Oshima, K. J. Am. Chem. Soc. 2003, 125, 1484.

[47] Masuda, K.; Sakiyama, N.; Tanaka, R.; Noguchi, K.; Tanaka, K. J. Am. Chem. Soc. 2011, 133, 6918. 